\title{
Downlink Cofrequency Interference Analysis of Vehicles and UAV Network in Ka Band
}

\author{
Maolin Wang, ${ }^{1,2}$ YuXin Zhang $\mathbb{D}^{3}$ and Zhi Wang ${ }^{1,2}$ \\ ${ }^{1}$ Zhe Jiang Key Laboratory of General Aviation Operation Technology, Jiande, China \\ ${ }^{2}$ CAAC Key Laboratory of General Aviation Operation, Department of General Aviation, \\ Civil Aviation Management Institute of China, Beijing, China \\ ${ }^{3}$ University of Science and Technology Beijing, Beijing, China \\ Correspondence should be addressed to YuXin Zhang; 1259829750@qq.com
}

Received 17 November 2021; Accepted 6 January 2022; Published 31 January 2022

Academic Editor: Renchao Xie

Copyright (c) 2022 Maolin Wang et al. This is an open access article distributed under the Creative Commons Attribution License, which permits unrestricted use, distribution, and reproduction in any medium, provided the original work is properly cited.

\begin{abstract}
With the rapid development of radio and computer technologies, the application scenarios of UAV carrying computer flight control systems are becoming more and more abundant. UAV can form a collaborative network with ground IoV (Internet of Vehicle) to improve the link quality of vehicle signals in some environments with poor network connectivity. For UAV and ground vehicles that are far apart, satellites can assist in the transmission of data between them. UAVs are allowed to be included in the satellite communication network in the Ka band in the 2015 World Radiocommunication Conference. In order to avoid the interference with existing communication services in the Ka band, it is necessary to limit the flying height of the UAV. This article will study the frequency compatibility between UAV and Internet of Vehicles in the same-frequency band. First, model the propagation environment of the UAV transmitting signal is modeled and the path loss of it is calculated, including the basic transmission loss in free space of the signal and the atmospheric propagation loss when the signal passes through the atmosphere. Then the radiation pattern of the receiving antenna is modeled, which is a $4 \times 2$ antenna array in this article, and the total antenna gain is obtained by adding the element gain and the array gain. Finally, the interference between UAV and Internet of Vehicles is simulated, calculating the interference and noise ratio of UAV to the Internet of Vehicles under different pointing angles between the interference and the interfered antenna and different elevation angles from UAV to the GSO satellite. Finally, the safe separation distance to protect the Internet of Vehicles from interference is got.
\end{abstract}

\section{Introduction}

Due to the mobility and rapid deployment of unmanned aerial vehicles, the application scenarios of UAVs are becoming more and more abundant in recent years, such as surveillance, disaster relief, and provision of cellular coverage [1-4]. The popularity of $5 \mathrm{G}$ technology has further accelerated the deployment process of UAVs in various applications [5-8]. UAVs can assist ground nodes in the air for data transmission, responsible for the relay and forwarding of transmitted data [9-11]. When a certain part of the Internet of Vehicles is facing a sudden increase in communication demand, the UAV can share part of the traffic, ensuring the communication demand for a certain period of time. UAV will be recovered after the emergency, effectively solving the problem of dynamic traffic load. In areas where natural disasters such as earthquakes occur, communication infrastructure is usually damaged and unable to work. UAV base stations can be deployed to provide communication services for rescue vehicles to ensure the smooth progress of rescue work. For remote areas on land or on the sea surface beyond the coverage of the ground vehicle network, satellites can help transmit data between UAVs and ground vehicles that are far apart. Therefore, satellite-enabled UAV communication is a promising development direction for future communication networks [12].

Although the UAV network has a lot of advantages, there are still many problems that have not been resolved, such as high data transmission pressure and shortage of working spectrum. As a rapidly developing new technology, the UAV 
network requires new terrestrial spectrum and satellite spectrum allocation to support the growth of its service. The increasing demand for spectrum has put tremendous pressure on existing services [13]. The 2015 World Radiocommunication Conference determined that UAVs are allowed to be included in the satellite communication network in the Ka band, so that communication links between UAVs and remote pilots can be established through the fixed satellite service network [14]. Since other services (such as Internet of Vehicles services) have already existed in the Ka frequency band, UAVs need to share this frequency band with existing services. Frequency reuse causes cofrequency interference. Moreover, because of the high altitude of UAV, the communication link between UAV and ground vehicles is often dominated by the line-of-sight channel, which causes serious downlink interference from UAVs to many adjacent but unrelated ground vehicles [15-17], greatly reducing the communication performance of the UAV in the downlink. Therefore, the cochannel interference of the downlink urgently needs to be studied.

\section{Materials and Methods}

As shown in Figure 1, considering the downlink communication of the UAV and IoV collaborative network in a specific area, the ground vehicle and UAV coexist in the same area. Due to frequency reuse, UAV and ground vehicles share the Ka frequency band, resulting in potential strong interference from UAV to vehicles through the downlink loss channel [18]. The link marked $\mathrm{x}$ is the interference path from the UAV to the ground vehicle. The UAV flies on a great circle path at a constant height $d$, equipped with a beamforming array antenna, the main beam axis of which points to the GSO FSS satellite. Depending on the relative position of UAV and satellite, the elevation angle to the satellite takes different values. The relative position relationship of the UAV, FSS satellite, and ground vehicle in the horizontal plane is shown in Figure 2. The relative azimuth angle between the UAV antenna and the vehicle antenna is $\varphi$. The downlink cofrequency interference will be analyzed in this article to impose restrictions on the operating parameters of the UAV, limiting the operation height of the UAV, so as to avoid serious interference to ground vehicles [19].

The interference caused by UAVs to the Internet of Vehicles is represented by the interference-to-noise ratio $(\mathrm{I} / \mathrm{N})$ [20], which is calculated by the following equation:

$$
\begin{aligned}
\frac{I}{N}= & P_{\mathrm{UAV}}-A_{\mathrm{UAV} \text { tovehicle }}-P_{f s}-P_{\text {Fuselage }}-P_{\text {atmosphere }} \\
& +G_{\text {vehicle }}-N_{\text {vehicle }},
\end{aligned}
$$

where $P_{\mathrm{UAV}}$ is the UAV antenna transmit power towards vehicles, and the value of $P_{\mathrm{UAV}}$ is determined, given in Table 1 [21].

The main axis of the UAV antenna points to the corresponding FSS satellite, and $\Theta$ is defined as the angle between the antenna main axis and the vector from UAV to ground vehicles, i.e., off-axis angle, which is more than two

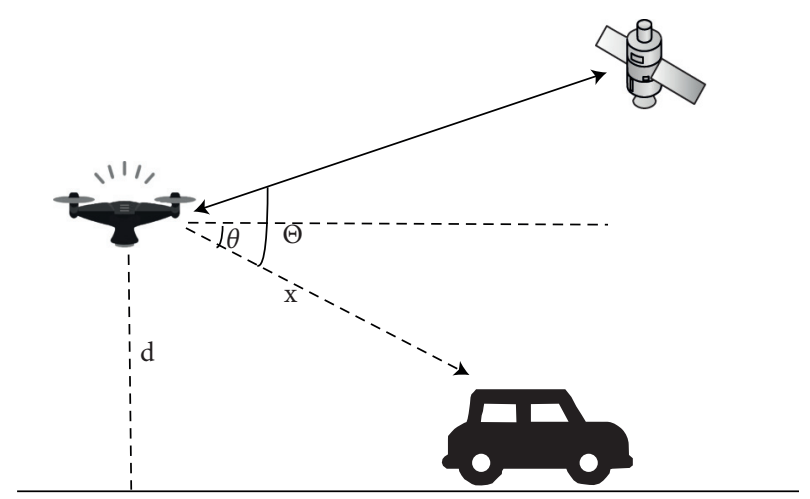

FIgUre 1: The positional relationship of UAVs, vehicles, and satellites in the vertical plane.

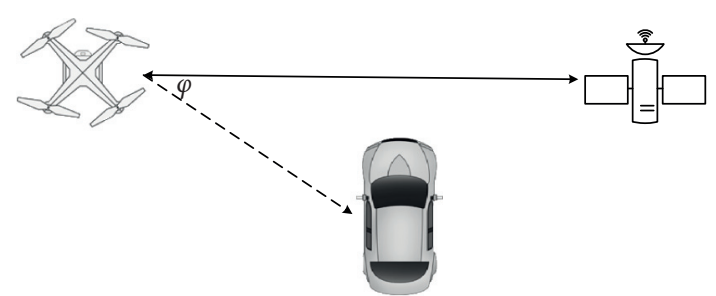

FIgURE 2: The positional relationship of UAVs, vehicles, and satellites in the horizontal plane.

TABLE 1: The EIRP restrictions that UAVs should comply with when operating in the Ka band.

\begin{tabular}{lc}
\hline Angle $\Theta$ & Maximum EIRP per $40 \mathrm{kHz}$ \\
\hline $2^{\circ} \leq \Theta \leq 7^{\circ}$ & $19-25 \log \Theta \mathrm{dB}(\mathrm{W} / 40 \mathrm{kHz})$ \\
$7^{\circ}<\Theta \leq 9.2^{\circ}$ & $-2 \mathrm{~dB}(\mathrm{~W} / 40 \mathrm{kHz})$ \\
$9.2^{\circ}<\Theta \leq 48^{\circ}$ & $22-25 \log \Theta \mathrm{dB}(\mathrm{W} / 40 \mathrm{kHz})$ \\
$48^{\circ}<\Theta \leq 180^{\circ}$ & $-10 \mathrm{~dB}(\mathrm{~W} / 40 \mathrm{kHz})$ \\
\hline
\end{tabular}

degrees, as shown in Figure 1. It should be noted that the values in the table are the maximum values under clear sky conditions, and the rainfall attenuation is not considered.

$A_{U A V}$ to vehicle is an adjustment parameter to unify the unit. In this example, it is equal to $10 \log (100 \mathrm{MHz} / 40 \mathrm{kHz})$. $P_{f s}$ is the free space basic transmission loss of the point-topoint link, calculated by the following equation [22].

$$
P_{f s}=32.4+20 \log f_{\mathrm{UAV}}+20 \log d_{\mathrm{UAV} \text { to vehicle, }}
$$

where $f_{\mathrm{UAV}}$ is the working frequency of UAV communication in $\mathrm{MHz}$, and $d_{\mathrm{UAV} \text { to vehicle }}$ is the distance in $\mathrm{km}$ between UAV and the interfered vehicle.

$P_{\text {atmosphere }}$ is the atmospheric attenuation, which is composed of the attenuation caused by dry air and water vapor. It can be obtained by the sum of oxygen and water vapor spectral lines when the pressure, temperature, and humidity of a certain place are known. The gas attenuation of the inclined path in the uplink between the heights $h_{1}$ and $h_{2}$ is calculated as follows $\left(h_{2}>h_{1} \geq 0 \mathrm{~km}\right)$ : 


$$
\begin{aligned}
A_{\text {gas }} & =\int_{h 1}^{h 2} \frac{\gamma(h)}{\sin \phi(h)} d h=\int_{h 1}^{h 2} \frac{\gamma(h)}{\sqrt{1-\cos ^{2} \phi(h)}} d h, \\
\cos \phi(h) & =\frac{\left(R_{E}+h_{1}\right) n\left(h_{1}\right)}{\left(R_{E}+h\right) n(h)} \cos \phi_{1},
\end{aligned}
$$

where $\gamma(h)$ is the specific attenuation at height $\mathrm{h}, R_{E}$ is the average earth radius (valued at $6371 \mathrm{~km}$ ), $\varphi_{1}$ is the apparent elevation angle at height $h_{1}$, and $n(h)$ is the refractive index at height $h$. Downlink attenuation and uplink attenuation are symmetrical.

The calculation formula for specific attenuation $\gamma(h)$ is as follows:

$$
\begin{aligned}
\gamma & =0.1820 f\left(N^{\prime \prime} \mathrm{Oxy}(f)+N^{\prime \prime} \text { water }(f)\right) \mathrm{dB} / \mathrm{km}, \\
N_{\text {Oxy }}^{\prime \prime}(f) & =\sum_{j(\text { Oxy })} S_{j} F_{j}+N_{D}^{\prime \prime}(f), \\
N_{\text {water }}^{\prime \prime}(f) & =\sum_{j(\text { water })} S_{j} F_{j},
\end{aligned}
$$

where $S_{j}$ is the intensity of the $j^{\text {th }}$ oxygen spectrum line or water vapor spectrum line:

$$
\begin{aligned}
S_{j} & =\alpha_{1} p\left(\frac{300}{T}\right)^{3} \exp \left[\alpha_{2}\left(1-\frac{300}{T}\right)\right] \times 10^{-7}, \quad \text { oxygen } \\
& =\beta_{1} e\left(\frac{300}{T}\right)^{3.5} \exp \left[\beta_{2}\left(1-\frac{300}{T}\right)\right] \times 10^{-1}, \quad \text { vapor, }
\end{aligned}
$$

where $p$ is the dry air pressure (hPa), $\alpha$ and $\beta$ are the spectral data of oxygen and water vapor, respectively, and $e$ is the partial pressure of water vapor $(\mathrm{hPa})$, which can be calculated from the water vapor density $\rho$ and temperature $T$ at a given height:

$$
e=\frac{\rho T}{216.7},
$$

where $T$ is the temperature $(\mathrm{k})$ and $F_{j}$ is the shape factor of the oxygen or water vapor line:

$$
F_{j}=\frac{f}{f_{j}}\left[\frac{\Delta f-\delta\left(f_{\mathrm{j}}-f\right)}{\left(f_{j}-f\right)^{2}+\Delta f^{2}} \frac{+\Delta f-\delta(f+f)}{\left(f_{j}+f\right)^{2}+\Delta f^{2}}\right],
$$

where $f_{j}$ is the line frequency of oxygen or water vapor, and $\Delta f$ is the line width:

$$
\begin{aligned}
\Delta f & =\alpha_{3} \times 10^{-4}\left(p \theta^{\left(0.8-\alpha_{4}\right)}+1.1 e \theta\right), \quad \text { oxygen } \\
& =\beta_{3} \times 10^{-4}\left(p \theta^{\beta_{4}}+\beta_{5} e \theta^{\beta_{6}}\right), \quad \text { vapor. }
\end{aligned}
$$

The calculation of the line width $\Delta f$ takes into account the Zeeman split of the oxygen line and the Doppler broadening of the water vapor line:

$$
\begin{aligned}
\Delta f & =\sqrt{\Delta f^{2}+2.25 \times 10^{-6}}, \quad \text { oyxgen } \\
& =0.535 \Delta f+\sqrt{0.217 \Delta f^{2}+\frac{2.1316 \times 10^{-12} f_{i}^{2}}{\theta}}, \quad \text { vapor. }
\end{aligned}
$$
lines:

$\delta$ is the correction factor for interference between oxygen

$$
\begin{aligned}
\delta & =\left(a_{5}+a_{6} \theta\right) \times 10^{-4}(p+e) \theta^{0.8}, \quad \text { oyxgen } \\
& =0, \quad \text { vapor. }
\end{aligned}
$$

$N_{D}^{\prime \prime}(f)$ is the continuous absorption spectrum of dry air resulted by the nitrogen attenuation caused by air pressure and the Debye spectrum, calculated as follows:

$N_{D}^{\prime \prime}(f)=f p \theta^{2}\left[\frac{6.14 \times 10^{-5}}{w\left[1+(f / w)^{2}\right]}+\frac{1.4 \times 10^{-12} p \theta^{1.5}}{1+1.9 \times 10^{-5} f^{1.5}}\right]$,

where $w$ is the width parameter of the Debye spectrum:

$$
w=5.6 \times 10^{-4} \theta^{0.8}(p+e) .
$$

$G_{\text {vehicle }}$ is the gain of the ground vehicle antenna towards the UAV. Ground mobile systems in the Ka frequency band usually use patch array antennas. Several identical patch elements are distributed on the $y-z$ plane at a fixed spacing distance $\lambda / 2$ [23-25], and the maximum gain is along the $\mathrm{x}$-axis direction. The arrow in Figure 3 indicates the signal transmission direction, the elevation angle is $\theta, 0^{\circ} \leq \theta \leq 180^{\circ}$, and the azimuth angle is $\varphi,-180^{\circ} \leq \varphi \leq 180^{\circ}$.

The radiation pattern of a single element in antenna array is calculated by the following equation:

$A_{\text {Ele }}(\phi, \theta)=G_{\text {Ele,max }}-\min \left\{-\left[A_{\text {Ele,Horizon }}(\phi)+A_{\text {Ele,Vertical }}(\theta)\right], A_{m}\right\}$.

Among them, the horizontal and vertical radiation patterns are

$$
\begin{aligned}
& A_{\text {Ele,Horizon }}(\phi)=-\min \left[12\left(\frac{\phi}{\phi_{3 d B}}\right)^{2}, S L A\right], \\
& A_{\text {Ele,Vertical }}(\theta)=-\min \left[12\left(\frac{\theta-90}{\theta_{3 d B}}\right)^{2}, S L A\right],
\end{aligned}
$$




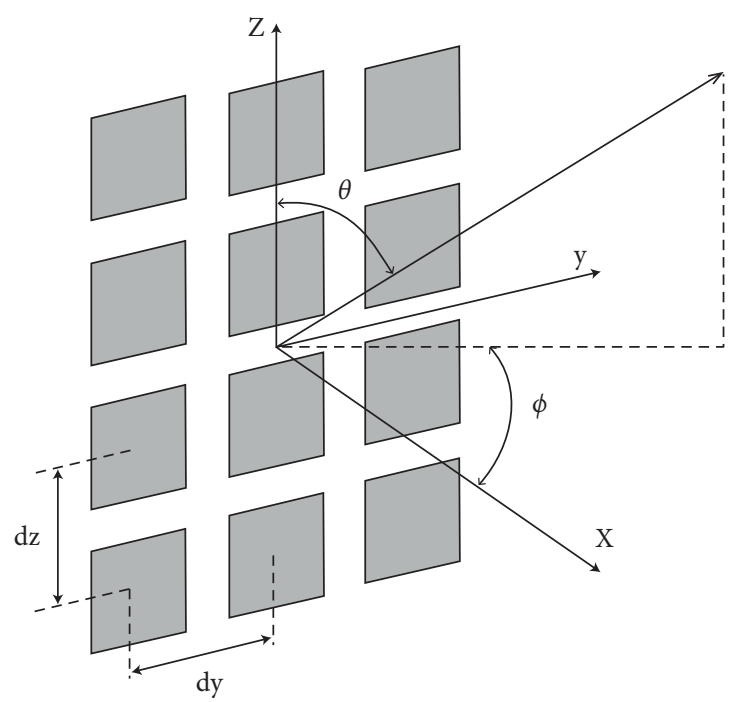

Figure 3: Antenna geometry model of the interfered system.

where $G_{E \text {,max }}$ is the peak gain of the array component, $S L A$ is the front-to-back ratio, and $\varphi_{3 d B}$ and $\theta_{3 d B}$ are the $3 \mathrm{~dB}$ beam widths.

The final radiation pattern of the beamforming array antenna is obtained by the following formula:

$$
A_{A}(\theta, \phi)=A_{E}(\theta, \phi)+10 \log _{10}\left(\left|\sum_{i=1}^{N_{H}} \sum_{j=1}^{N_{V}} u_{i, j} \cdot v_{i, j}\right|^{2}\right),
$$

where

$$
\begin{aligned}
& v_{i, j}=e \wedge\left(\sqrt{-1} \cdot 2 \pi\left((i-1) \frac{d_{V}}{\lambda} \cos \theta+(j-1) \frac{d_{H}}{\lambda} \sin \theta \sin \phi\right)\right), \\
& u_{i, j}=\frac{1}{\sqrt{N_{H} N_{V}}} e \wedge\left(\sqrt{-1} \cdot 2 \pi\left[(i-1) \frac{d_{V}}{\lambda} \sin \theta-(j-1) \frac{d_{H}}{\lambda} \cos \theta \sin (90-\phi)\right]\right),
\end{aligned}
$$

where $N_{V}$ and $N_{H}$ are the number of elements, and $d_{V}$ and $d_{H}$ are the separation distances of the elements in the vertical and horizontal directions of the antenna array, respectively.

The last term $N_{\text {vehicle }}$ in (1) is the total noise at vehicles:

$$
N_{\text {vehicle }}=10 \log _{10}(k B T)+N_{f},
$$

where $k$ is Boltzmann's constant, $B$ is the working bandwidth of vehicles, $T$ is the thermodynamic temperature, and $N_{f}$ is the noise figure.

\section{Results and Discussion}

Simulation results of cochannel interference between UAVs and vehicles is provided in this section to analyze the downlink interference situation of the UAV network. The simulation parameters are given in Table 2. Consider the situation where there is only one UAV interference source within the line of sight of the vehicle in a specific area at a specific time. The vehicle antenna height is $1.5 \mathrm{~m}$. The acceptable interference level threshold for the Internet of Vehicles service at $28 \mathrm{GHz}$ is $-6 \mathrm{~dB}$ [26]. The UAV network has a working bandwidth of $100 \mathrm{MHz}$. In order to fully analyze different interference scenarios, consider the following two cases:

(1) The antennas of the vehicle and the UAV are directed to each other with maximum gain, and the downlink interference between the UAV network and the Internet of Vehicles service is analyzed under different relative positions between the UAV and the satellite. In this case, the elevation angles from the UAV to the satellite are $5^{\circ}, 45^{\circ}$, and $70^{\circ}$, and the antenna gains of the UAV and the vehicle are both peak gains, that is, $\theta=90^{\circ}, \varphi=180^{\circ}$ in (15).

(2) The elevation angle from UAV to the satellite is fixed to $10^{\circ}$, and the interference between the UAV and the vehicle is analyzed at different antenna relative points. In this case, it is assumed that the vehicle antenna has a mechanical downtilt angle of $10^{\circ}$ [27] and a maximum of $6^{\circ}$ [28] electronic uptilt. The antenna of the user end keeps pointing horizontally, and the azimuth angle between the UAV and the vehicle antenna is $5^{\circ}, 30^{\circ}$, and $180^{\circ}$.

The simulation result of case 1 is shown in Figures 4-6. The 3 curves of blue, green, and yellow, respectively, 
TABLE 2: Simulation parameters in this study.

\begin{tabular}{lccc}
\hline Parameter & Value & Parameter & Value \\
\hline GE, max & $5 \mathrm{dBi}$ & $\varphi 3 \mathrm{~dB}$ & $80^{\circ}$ \\
SLA & 30 & $\theta 3 \mathrm{~dB}$ & $65^{\circ}$ \\
NV & 4 & $\mathrm{NH}$ & 2 \\
dV & $0.5 \lambda$ & $\mathrm{dH}$ & $0.5 \lambda$ \\
\hline
\end{tabular}

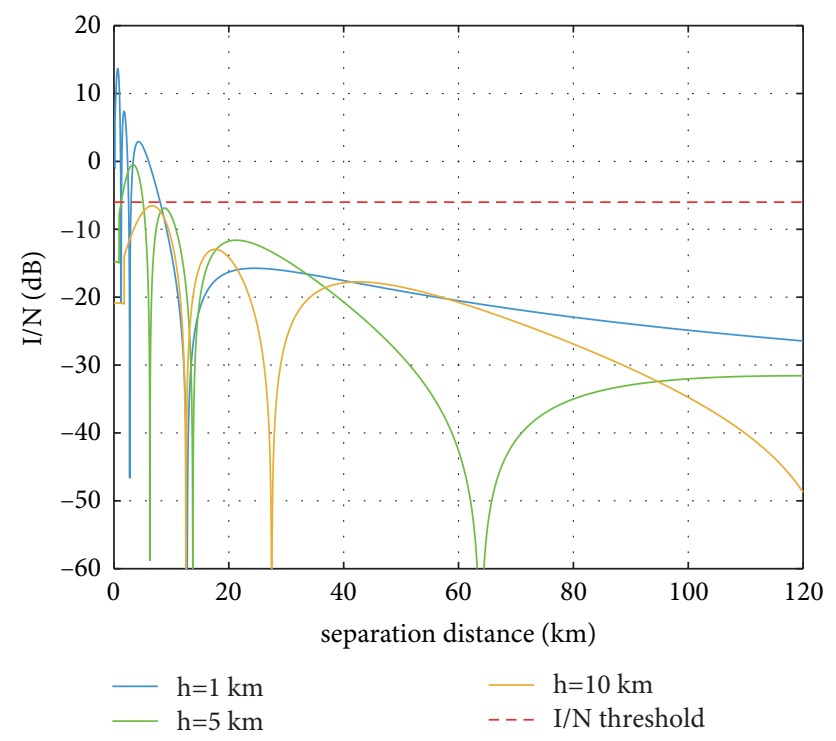

Figure 4: Case 1, when the vehicle is interfered at an elevation angle of $5^{\circ}$.

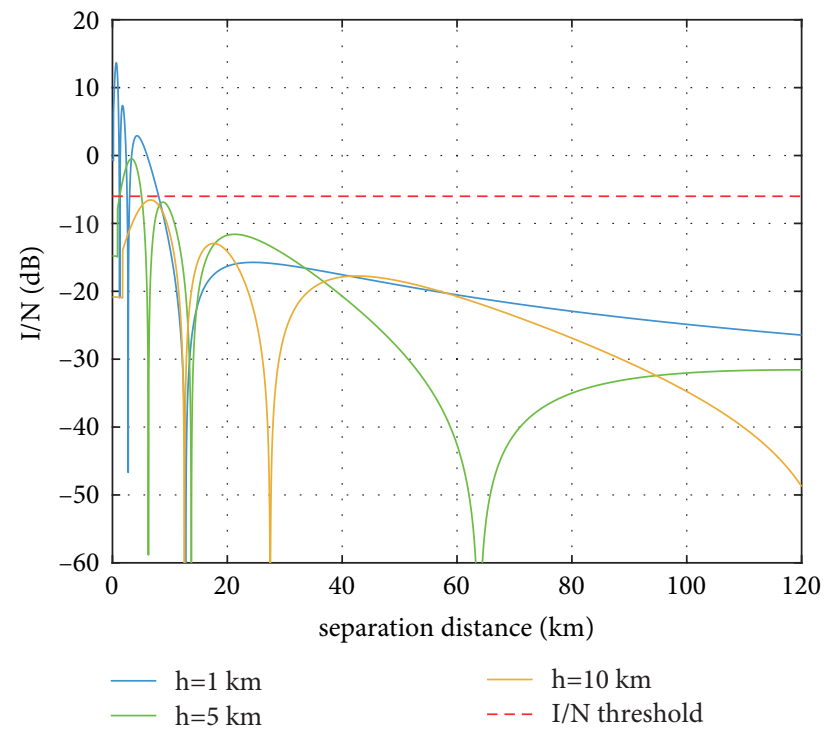

Figure 5: Case 1, when the vehicle is interfered at an elevation angle of $45^{\circ}$.

represent the three flying heights 1,5 , and $10 \mathrm{~km}$ of UAV, and the red horizontal line represents the acceptable interference threshold of ground vehicles. The abscissa is the distance between UAV and vehicle in the horizontal direction, and the ordinate is the level of interference caused by the UAV to ground vehicles. The abscissa corresponding to the intersection of the interference curve and the horizontal line is the minimum separation distance required to protect the ground vehicle network from interference.

It can be seen that as the flying height of UAVs increases, the interference level of UAVs to ground vehicles gradually decreases, and the required separation distance between the 


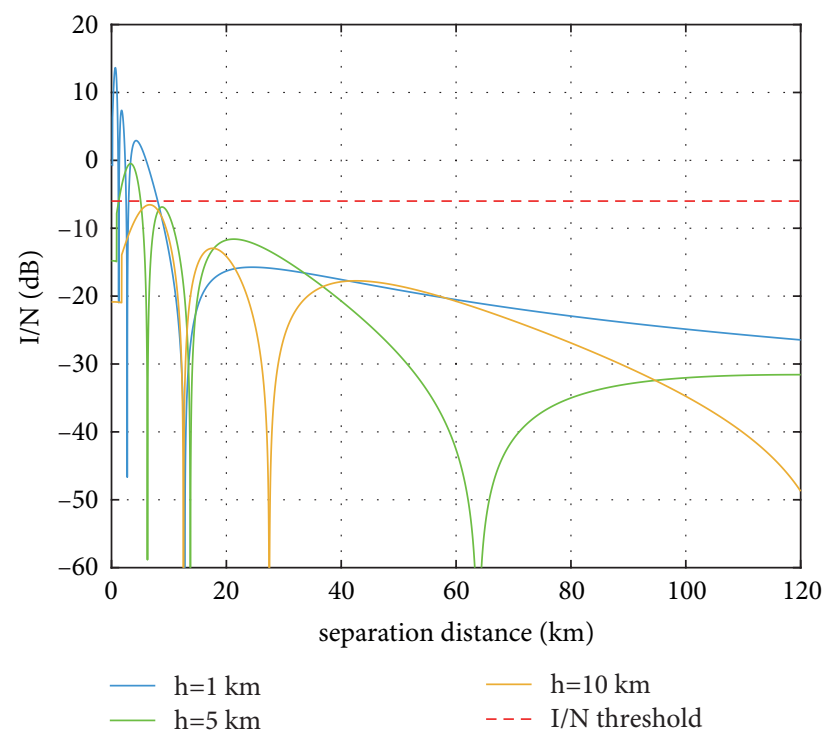

Figure 6: Case 1, when the vehicle is interfered at an elevation angle of $70^{\circ}$.

two is getting smaller and smaller. When the flying height is $10 \mathrm{~km}$, the interference curve keeps below the threshold. In this situation, even if the UAV is directly above the vehicle, it will not cause unacceptable interference to the vehicle. The envelope of the interference curve decreases as the distance increases, which is consistent with experience, because the interference signal will attenuate in the propagation path. The fluctuations of the interference curve are caused by the radiation pattern of the receiving antenna. In the radiation pattern, the antenna gain continuously fluctuates with the change of elevation and azimuth. While the altitude difference of UAV and vehicle antenna keeps the same, the relative elevation angle between the antennas will change with the separation distance, so the interference curve also has ups and downs.

In order to express the simulation results more clearly, read the abscissa value of the intersection of each curve and the threshold line and fill in the table, as given in Table 3.

It can be seen that when the elevation angles of the UAV relative to the satellite are $5^{\circ}, 45^{\circ}$, and $70^{\circ}$, there is no significant difference in the minimum separation distance between the UAV and the vehicle. Therefore, the elevation angle between the UAV and the satellite, that is, the relative position relationship between the two, has no significant impact on the level of interference between the UAV and the Internet of Vehicles and can be ignored in the interference analysis. Since the case 1 assumes that the vehicle and the UAV antenna keep the maximum gain pointing to each other, the interference level simulating this time is actually the maximum possible value, and it is almost impossible to reach the maximum value in reality. In order to get a simulation result closer to the actual situation, continue with the simulation of case 2 .

The simulation results of case 2 are shown in Figures 7-9. It can be clearly seen that the greater the azimuth angle between the transmitting antenna and the receiving antenna, the lower the level of interference that
TABLE 3: The safe separation distance between UAV and the vehicle in case 1 .

\begin{tabular}{lcccc}
\hline UAV height $(\mathrm{km})$ & & \multicolumn{3}{c}{ Minimum separation } \\
& & \multicolumn{3}{c}{ distance $(\mathrm{km})$} \\
& & 1 & 5 & 10 \\
\hline \multirow{3}{*}{ UAV elevation angle $\left(^{\circ}\right)$} & 45 & 8.1 & 5.06 & 0 \\
& 70 & 8.02 & 5.1 & 0 \\
& & & 4.92 & 0 \\
\hline
\end{tabular}

the vehicle will receive, and the shorter the separation distance between the UAV and the vehicle needs to keep. When the relative azimuth angle is $180^{\circ}$, i.e., the main axis of receiving antenna and transmitting antenna beams deviate from each other, the interference from $\mathrm{UAV}$ to the Internet of Vehicles is almost negligible, and there is no need to impose restrictions on the flying height of the UAV. This is because the antenna peak gain is at the azimuth angle of $0^{\circ}$, and the further away from $0^{\circ}$, the smaller the gain, so the interference received by the interfered vehicle is also lower. The abscissa value of the intersection of each interference curve and the threshold line is given in Table 4 .

It can be seen from the table that the larger the azimuth angle between the antennas of the UAV and the ground vehicle, the shorter the separation distance between the two needs to be maintained. When the azimuth angle is $5^{\circ}$, the separation distance value is close to the simulation result of case 1 because the antenna gain decays slowly near the peak. When the azimuth angle is $30^{\circ}$, the separation distance value at different flying altitudes has been reduced by about $1 \mathrm{~km}$. Combining Tables 3 and 4, we can see that when the acceptable interference threshold of the Internet of Vehicles is $-6 \mathrm{~dB}$; in order to protect the Internet of Vehicles from interference, the flying height of the UAV needs to be limited to more than $10 \mathrm{~km}$, while the horizontal distance between 


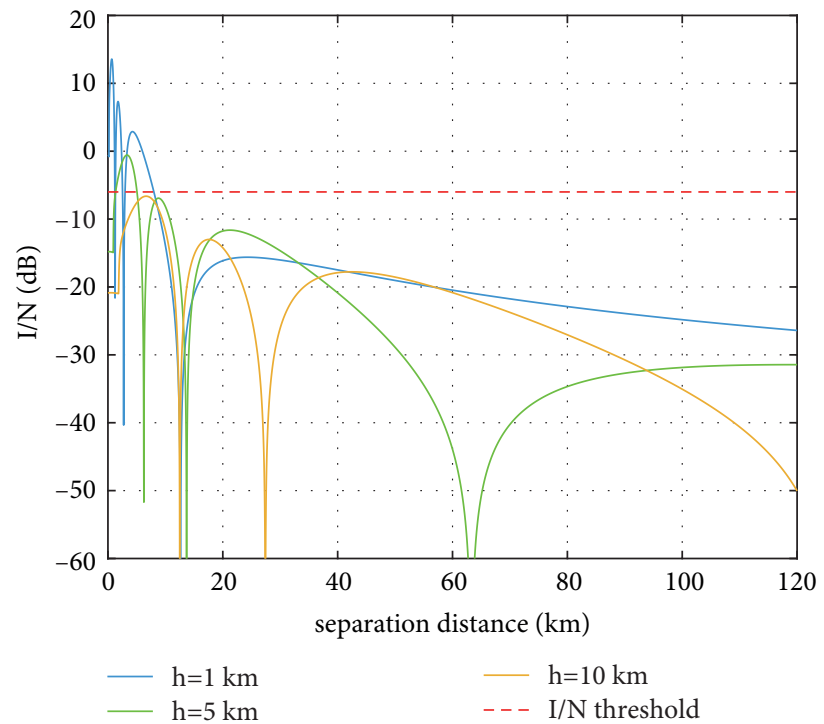

FIgURE 7: Case 2, when the vehicle is interfered at an azimuth angle of $5^{\circ}$.

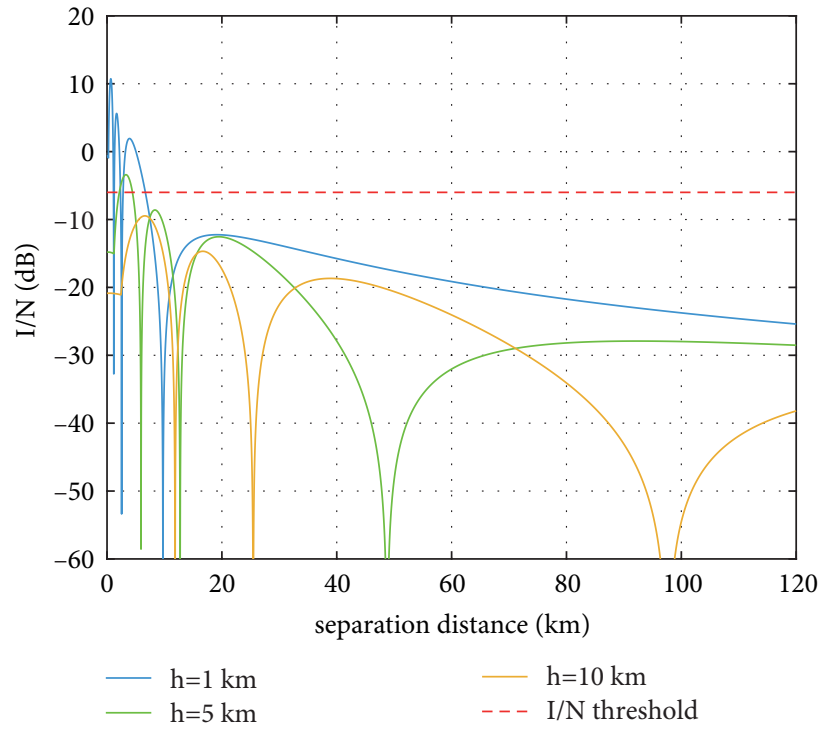

FIGURE 8: Case 2, when the vehicle is interfered at an azimuth angle of $30^{\circ}$.

the UAV and the ground vehicle is not restricted. It should be noted that this simulation is a static simulation, and the duration of interference and the possibility of interference are not taken into consideration in the calculation process. To obtain simulation results closer to the actual situation, the above two factors need to be considered. 


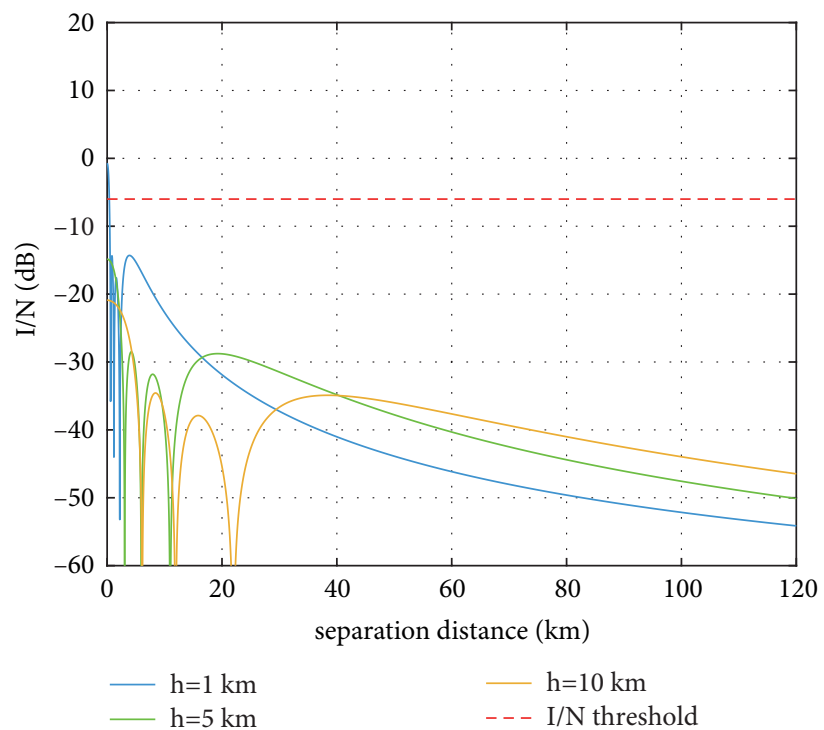

FIgURE 9: Case 2, when the vehicle is interfered at an azimuth angle of $180^{\circ}$.

TABLE 4: The safe separation distance between the UAV and the vehicle in case 2.

\begin{tabular}{|c|c|c|c|c|}
\hline \multirow{2}{*}{ UAV height $(\mathrm{km})$} & & \multicolumn{3}{|c|}{ Minimum separation distance $(\mathrm{km})$} \\
\hline & & 1 & 5 & 10 \\
\hline \multirow{3}{*}{ Azimuth angle between UAV and vehicle } & 5 & 8.06 & 5.14 & 0 \\
\hline & 30 & 6.9 & 4.28 & 0 \\
\hline & 180 & 0.48 & 0 & 0 \\
\hline
\end{tabular}

\section{Conclusion}

In this study, a simulation analysis of the same-frequency interference between UAVs and the Internet of Vehicles working in the Ka band was done to calculate the separation distance required to protect the Internet of Vehicles from UAV interference. When calculating the cofrequency interference, the propagation environment of the radio signal was modeled. The UAV transmitting signal experienced free space loss and atmospheric loss during the propagation process and finally became an interference signal after amplified by the receiving antenna. The receiving antenna model was a $4 \times 2$ antenna array. Two cases were considered in the calculation. One was the worst case, i.e., the UAV antenna and the vehicle antenna kept pointing to each other with maximum gain, and the azimuth angle was fixed at $0^{\circ}$. The second was the general case, i.e., the antenna gain would vary with the change of the azimuth angle. For the worst case, the elevation angle from the UAV to the GSO satellite was set to 3 different values to study the interference between the UAV and the vehicle at different relative positions between the UAV and satellite. For the general case, the azimuth angle between the UAV antenna and the vehicle antenna was set to 3 different values to study the interference of the 2 antennas at different mutual pointing angles. The safe separation distance calculated finally between the UAV and the Internet of Vehicles was summarized in the table. In actual situations, different standards could be selected according to the specific local conditions.

\section{Data Availability}

The data used to support the findings of this study are included within the article.

\section{Conflicts of Interest}

The authors declare that they have no conflicts of interest.

\section{References}

[1] Y. Zeng, J. Lyu, and R. Zhang, "Cellular-connected UAV: potential, challenges, and promising technologies," IEEE Wireless Communications, vol. 26, no. 1, pp. 120-127, 2019.

[2] A. Al-Hourani and K. Gomez, "Modeling cellular-to-UAV path-loss for suburban environments," IEEE Wireless Communications Letters, vol. 7, no. 1, pp. 82-85, 2018.

[3] M. M. Azari, G. Geraci, A. Garcia-Rodriguez, and S. Pollin, "UAV-to-UAV communications in cellular networks," IEEE Transactions on Wireless Communications, vol. 19, no. 9, pp. 6130-6144, 2020.

[4] S. Zhang, Y. Zeng, and R. Zhang, "Cellular-enabled UAV communication: a connectivity-constrained trajectory optimization perspective," IEEE Transactions on Communications, vol. 67, no. 3, pp. 2580-2604, 2019.

[5] S. Singh, H. Narasimhan, Güvenç, and A. Bhuyan, "Coverage analysis for ground and aerial users in mmWave cellular networks in urban settings," in Proceedings of the 2020 SoutheastCon, pp. 1-8, North Carolina, NC, USA, March 2020. 
[6] S. Zhang, "Research on emergency coverage capability of fixed-wing UAV based on 5G," in Proceedings of the 2020 International Wireless Communications and Mobile Computing (IWCMC), pp. 680-684, Limassol, Cyprus, June 2020.

[7] K. Yan, L. Ma, and Y. Zhang, "Research on the application of 5G technology in UAV data link," in Proceedings of the 2020 IEEE 9th Joint International Information Technology and Artificial Intelligence Conference (ITAIC), pp. 1115-1118, Chongqing, China, December 2020.

[8] B. Li, Z. Fei, and Y. Zhang, "UAV communications for 5G and beyond: recent advances and future trends," IEEE Internet of Things Journal, vol. 6, no. 2, pp. 2241-2263, 2019.

[9] L. Zhang, H. Zhao, S. Hou et al., "A survey on 5 G millimeter wave communications for UAV-assisted wireless networks," IEEE Access, vol. 7, pp. 117460-117504, 2019.

[10] S. K. Khan, "UAV-aided 5 G network in suburban, urban, dense urban, and high-rise urban environments," in Proceedings of the 2020 IEEE 19th International Symposium on Network Computing and Applications (NCA), pp. 1-4, Maryland, MA, USA, November 2020.

[11] L. Zhu, H. Liang, H. Wang, and B. Ning, "Joint security and train control design in blockchain empowered CBTC system," IEEE Internet of Things Journal, 2020.

[12] Y. Zeng, Q. Wu, and R. Zhang, "Accessing from the sky: a tutorial on UAV communications for 5G and beyond," Proceedings of the IEEE, vol. 107, no. 12, pp. 2327-2375, 2019.

[13] R. J. Kerczewski and L. Jonasson, "Outcomes of the 2015 world radiocommunication conference for aeronautical spectrum and applications," in Proceedings of the 2016 Integrated Communications Navigation and Surveillance (ICNS), Virginia, VA, USA, April 2016.

[14] ITU, Use of the Frequency Bands 19.7-20.2 GHz and 29.5-30 $\mathrm{GHz}$ by Earth Stations in Motion Communicating with Geostationary Space Stations in the Fixed-Satellite Service, Document Resolution 156, World Radiocommunication Conference, Geneva, Switzerland, 2015.

[15] W. Mei and R. Zhang, "Cooperative downlink interference transmission and cancellation for cellular-connected UAV: a divide-and-conquer approach," IEEE Transactions on Communications, vol. 68, no. 2, pp. 1297-1311, 2020.

[16] L. Zhu, Y. Li, F. R. Yu, B. Ning, T. Tang, and X. Wang, "Crosslayer defense methods for jamming-resistant CBTC systems," IEEE Transactions on Intelligent Transportation Systems, vol. 22, no. 11, pp. 7266-7278, 2021.

[17] Y. Li, L. Zhu, H. Wang, F. R. Yu, and S. Liu, "A cross-layer defense scheme for edge intelligence-enabled CBTC systems against MitM attacks," IEEE Transactions on Intelligent Transportation Systems, vol. 22, no. 4, pp. 2286-2298, 2021.

[18] S. P. Winter and A. Knopp, "Statistics of terrestrial fixed service interference in the aeronautical SATCOM channel," in Proceedings of the ICC 2019-2019 IEEE International Conference on Communications (ICC), pp. 1-7, Shanghai, China, 2019.

[19] C. P. Cerasoli, "Ka band spectrum re-use for surrogate satellites on unmanned airborne vehicles," in Proceedings of the MILCOM 2005-2005 IEEE Military Communications Conference, vol. 3, pp. 1977-1983, New Jersey, NJ, USA, October 2005.

[20] B. Kerczewski and B. Bishop, "UAS satellite earth station emission limits for terrestrial system interference protection," in Proceedings of the 2017 Integrated Communications, Navigation and Surveillance Conference (ICNS), pp. 1-17, Virginia, VA, USA, April 2017.
[21] E. E. Ahiagbe, D. K. Tettey, and H. Jo, "Methods to evaluate and mitigate the interference from maritime ESIM to other services in 27.5-29.5 GHz band," in Proceedings of the 2018 International Conference on Information and Communication Technology Convergence (ICTC), pp. 1058-1063, Jeju, South Korea, October 2018.

[22] International Telecommunication Union, The Concept Of Transmission Loss For Radio Links, ITU-R P.341, ITU, Geneva, Switzerland, 2019.

[23] H. -K. Kim, Y. Cho, and H. -S. Jo, “Adjacent channel compatibility evaluation and interference mitigation technique between earth station in motion and IMT-2020," IEEE Access, vol. 8, pp. 213185-213205, 2020.

[24] Y. Cho, K. Kim, M. Nekovee, and H.-S. Jo, "Coexistence of 5G with satellite services in the millimeter-wave band," IEEE Access, vol. 8, pp. 163618-163636, 2020.

[25] W. A. Hassan, H.-S. Jo, S. Ikki, and M. Nekovee, "Spectrumsharing method for co-existence between 5G OFDM-based system and fixed service," IEEE Access, vol. 7, pp. 77460-77475, 2019.

[26] ITU, Annex 21 to Document 5A/469-E, World Radiocommunication Conference, Geneva, Switzerland, 2017.

[27] D. Oh and J. Park, "Protection of the mobile station from the interference by maritime earth station in motion in the 28 $\mathrm{GHz}$ band," in Proceedings of the 36th International Communications Satellite Systems Conference (ICSSC 2018), pp. 1-4, Niagra falls, Canada, October 2018.

[28] ITU, Document 4A/885-E[C], World Radiocommunication Conference, Geneva, 2019. 